\title{
The Numerical Solution of Two-Dimensional Volterra Integral Equations by Collocation and Iterated Collocation
}

\author{
H. Brunner \\ Department of Mathematics and Statistics, Memorial University of Newfoundland, \\ St John's, Newfoundland, Canada A1C 5S7 \\ AND J.-P. KAUTHEN \\ Institut de Mathématiques, Université de Fribourg, CH-1700 Fribourg, \\ Switzerland
}

[Received 6 May 1988]

\begin{abstract}
While the numerical solution of one-dimensional Volterra integral equations of the second kind with regular kernels is now well understood there exist no systematic studies of the approximate solution of their two-dimensional counterparts. In the present paper we analyse the numerical solution of such equations by methods based on collocation and iterated collocation techniques in certain polynomial spline spaces. The analysis focuses on the global convergence and local superconvergence properties of the approximating spline functions.
\end{abstract}

\section{Introduction}

LET $D:=[0, X] \times[0, Y] \subset \mathbb{R}^{2}$ be a given rectangular domain and consider the Volterra integral equation of the second kind

$$
f(x, y)=g(x, y)+\int_{0}^{x} \int_{0}^{y} K(x, y, \xi, \eta) f(\xi, \eta) \mathrm{d} \xi \mathrm{d} \eta, \quad(x, y) \in D .
$$

Here, $g$ and $K$ are given (real-valued) continuous functions defined, respectively, on $D$ and $S:=\{(x, y, \xi, \eta): 0 \leqslant \xi \leqslant x \leqslant X, 0 \leqslant \eta \leqslant y \leqslant Y\}$. It follows from the classical theory of Volterra (see, for example, $[18,11,10])$ that $(1.1)$ possesses a unique solution $f \in C(D)$.

During the last ten years significant progress has been made in the numerical analysis of the one-dimensional version of (1.1) (see for example, [4,5]). However, there are no studies dealing with the derivation and analysis of general numerical methods for the two-dimensional integral equation (1.1) (in contrast to two-dimensional Fredholm integral equations; compare $[8,9])$. Of the few papers concerned with specific methods for (1.1) we mention $[2,16]$ : the former introduces a class of explicit Runge-Kutta-type methods of order 3 (without analysing their convergence), and the latter employs bivariate cubic spline functions of full continuity.

In the present paper we introduce collocation and iterated collocation methods for (1.1) and give an analysis of the global and local convergence properties of the 
approximating polynomial spline function. The approach used here may be viewed as a generalization of the one used in [3] for one-dimensional Volterra integral equations of the second kind. The paper is organized as follows. In Section 2 we describe the approximating spline spaces and derive results on the attainable orders of global convergence and local superconvergence. Section 3 deals with iterated collocation approximations and their local superconvergence properties. Since in most realistic applications the integrals occurring in the collocation equations cannot be found analytically, a further discretization step, involving suitable quadrature processes, will be necessary. This will be discussed and illustrated numerically in Section 4. The paper concludes with a brief section dealing with an application of these methods to certain hyperbolic initial-value problems.

\section{Collocation using polynomial spline functions}

\subsection{The Approximating Polynomial Spline Spaces}

Let $\Pi_{M}^{(1)}$ and $\Pi_{N}^{(2)}$ denote, respectively, partitions of the intervals $[0, X]$ and $[0, Y]$ in $D=[0, X] \times[0, Y]$,

$$
\Pi_{M}^{(1)}: 0=x_{0}<x_{1}<\cdots<x_{M}=X \quad\left(\text { where } x_{m}=x_{m}^{(M)}\right),
$$

and

$$
\Pi_{N}^{(2)}: 0=y_{0}<y_{1}<\cdots<y_{N}=Y \quad\left(y_{n}=y_{n}^{(N)}\right) .
$$

These partitions define a grid for $D$,

$$
\Pi_{M, N}:=\Pi_{M}^{(1)} \times \Pi_{N}^{(2)}=\left\{\left(x_{m}, y_{n}\right): 0 \leqslant m \leqslant M, 0 \leqslant n \leqslant N\right\}
$$

Set

$$
\begin{array}{ccl}
\sigma_{0}^{(1)}:=\left[x_{0}, x_{1}\right], & \sigma_{m}^{(1)}:=\left(x_{m}, x_{m+1}\right] & (m=1, \ldots, M-1), \\
\sigma_{0}^{(2)}:=\left[y_{0}, y_{1}\right], & \sigma_{n}^{(2)}:=\left(y_{n}, y_{n+1}\right] & (n=1, \ldots, N-1),
\end{array}
$$

and

$$
D_{m, n}:=\sigma_{m}^{(1)} \times \sigma_{n}^{(2)} \quad(m=0, \ldots, M-1 ; n=0, \ldots, N-1) .
$$

If, for given positive integers $p$ and $q, \pi_{p-1, q-1}$ denotes the space of real polynomials of degree $p-1$ in $x$ and degree $q-1$ in $y$, then

$$
S_{p, q}^{(-1)}\left(\Pi_{M, N}\right):=\left\{u:\left.u\right|_{D_{m, n}}:=u_{m, n} \in \pi_{p-1, q-1}, 0 \leqslant m \leqslant M-1,0 \leqslant n \leqslant N-1\right\},
$$

is the space of bivariate polynomial spline functions of order $p$ (degree $p-1)$ in $x$ and order $q$ (degree $q-1$ ) in $y$. An element of this space has, in general, jump discontinuities at the interior grid lines $x=x_{m}(m=1, \ldots, M-1)$ and $y=y_{n}$ $(n=1, \ldots, N-1)$. The dimension of this linear space is easily seen to be $M N p q$ (note that $S_{p, q}^{(-1)}\left(\Pi_{M, N}\right)$ is a tensor-product space based on the univariate spline spaces $S_{p}^{(-1)}\left(\Pi_{M}^{(1)}\right)$ and $\left.S_{q}^{(-1)}\left(\Pi_{N}^{(2)}\right)\right)$. 
On a rectangle $D_{m, n}$ defined by the grid $\Pi_{M, N}$ the element $u \in S_{p, q}^{(-1)}\left(\Pi_{M, N}\right)$ is given by

$$
u_{m, n}(x, y)=\sum_{k=1}^{p} \sum_{l=1}^{q} \alpha \alpha_{k, l}^{(m, n)} \tau^{k-1} \theta^{i-1}
$$

where we have set $x=x_{m}+\tau h_{m}^{(1)}, y=y_{n}+\theta h_{n}^{(2)}$, with

$$
h_{m}^{(1)}:=x_{m+1}-x_{m}, \quad h_{n}^{(2)}:=y_{n+1}-y_{n} \text {; }
$$

here, the coefficients $\alpha_{k, I}^{(m, n)}$ depend on $h_{m}^{(1)}$ and $h_{n}^{(2)}$. Instead of (2.2) we shall frequently use the Lagrange representation of $u$ on $D_{m, n}$,

$$
u_{m, n}(x, y)=\sum_{k=1}^{p} \sum_{i=1}^{q} L_{k, l}(\tau, \theta) Y Y_{k, l}^{(m, n)} .
$$

Here, $Y_{k, l}^{(m, n)}:=u_{m, n}\left(x_{m}+c_{k} h_{m}^{(1)}, y_{n}+d_{l} h_{n}^{(2)}\right)$ and $L_{k, l}(\tau, \theta):=L_{k}^{(1)}(\tau) L l^{(2)}(\theta)$, with

$$
L k_{k}^{(1)}(\tau):=\prod_{\substack{r=1 \\ r \neq k}}^{p}\left(\tau-c_{r}\right) /\left(c_{k}-c_{r}\right), \quad L l^{(2)}(\theta):=\prod_{\substack{s=1 \\ s \neq l}}^{q}\left(\theta-d_{s}\right) /\left(d_{l}-d_{s}\right)
$$

denoting, respectively, the Lagrange fundamental polynomials corresponding to given parameters $0 \leqslant c_{1}<\cdots<c_{p} \leqslant 1,0 \leqslant d_{1}<\cdots<d_{q} \leqslant 1$.

A discussion of general tensor-product spline spaces may be found, for example, in $[13,14]$.

\subsection{Collocation Approximation in $S_{p, q}^{(-1)}\left(\Pi_{M, N}\right)$}

The solution $f$ of the integral equation (1.1) will be approximated by an element $u$ of the spline space $S_{p, q}^{(-1)}\left(\Pi_{M, N}\right)$. In ordeI to set up (and, also, to motivate) the collocation equation defining $u$ we first rewrite (1.1) in a form related to the given grid $\Pi_{M, N}$ of $D$. If

$$
(x, y)=\left(x_{m}+\tau h_{m}^{(1)}, y_{n}+\theta h_{n}^{(2)}\right) \in D_{m, n},
$$

then (1.1) may be written as

$$
\begin{aligned}
f(x, y)= & g(x, y)+h_{m}^{(1)} h_{n}^{(2)} \\
& \times \int_{0}^{\tau} \int_{0}^{\theta} K\left(x, y, x_{m}(s), y_{n}(t)\right) f\left(x_{m}(s), y_{n}(t)\right) \mathrm{d} s \mathrm{~d} t+F_{m, n}(x, y ; f) .
\end{aligned}
$$

Here, we have used the notation $x_{m}(s):=x_{m}+s h_{m}^{(1)}, y_{n}(t):=y_{n}+t h_{n}^{(2)}$; the lag term $F_{m, n}$ is given by

$$
\begin{aligned}
F_{m, n}(x, y, f):= & h_{n}^{(1)} \sum_{v=0}^{n-1} h_{v}^{(2)} \int_{0}^{\tau} \int_{0}^{1} K\left(x, y, x_{m}(s), y_{v}(t)\right) f\left(x_{m}(s), y_{v}(t)\right) \mathrm{d} s \mathrm{~d} t \\
& +h_{n}^{(2)} \sum_{\mu=0}^{m-1} h_{\mu}^{(1)} \int_{0}^{1} \int_{0}^{\theta} K\left(x, y, x_{\mu}(s), y_{n}(t)\right) f\left(x_{\mu}(s), y_{n}(t)\right) \mathrm{d} s \mathrm{~d} t \\
& +\sum_{\mu=0}^{m-1} \sum_{v=0}^{n-1} h_{\mu}^{(1)} h_{v}^{(2)} \int_{0}^{1} \int_{0}^{1} K\left(x, y, x_{\mu}(s), y_{v}(t)\right) f\left(x_{\mu}(s), y_{v}(t)\right) \mathrm{d} s \mathrm{~d} t
\end{aligned}
$$


(Here, if $m=0$ or $n=0$ the corresponding sum is to be taken as zero.) Note that the solution $f$ of (1.1) is known on part of the boundary of $D$ :

$$
f(x, y)= \begin{cases}g(x, 0) & \text { if } 0 \leqslant x \leqslant X \text { and } y=0 \\ g(0, y) & \text { if } 0 \leqslant y \leqslant Y \text { and } x=0\end{cases}
$$

Introducing the sets

$$
X_{m}:=\left\{x=x_{m, i}:=x_{m}+c_{i} h_{m}^{(1)}: 0 \leqslant c_{1}<\cdots<c_{p} \leqslant 1\right\},
$$

$(m=0, \ldots, M-1)$, and

$$
Y_{n}:=\left\{y=y_{n, j}:=y_{n}+d_{j} h_{n}^{(2)}: 0 \leqslant d_{1}<\cdots<d_{q} \leqslant 1\right\},
$$

$(n=0, \ldots, N-1)$, where the $\left\{c_{i}\right\}$ and $\left\{d_{j}\right\}$ are given real numbers (the collocation parameters), we define the set of collocation points by

$$
C(M, N):=\bigcup_{(m, n)} C_{m, n} \subset D, \quad \text { with } C_{m, n}:=X_{m} \times Y_{n} .
$$

The desired approximation $u \in S_{p, q}^{(-1)}\left(\Pi_{M, N}\right)$ is to satisfy the integral equation (1.1) on the finite subset $C(M, N)$ of $D$. Thus, according to (2.4), the collocation equation describing this requirement on $D_{m, n}$ has the form

$$
\begin{aligned}
u_{m, n}\left(x_{m, i}, y_{n, j}\right)= & g\left(x_{m, i}, y_{n, j}\right) \\
& +h_{m}^{(1)} h_{n}^{(2)} \int_{0}^{c_{1}} \int_{0}^{d_{j}} K\left(x_{m, i}, y_{n, j}, x_{m}(s), y_{n}(t)\right) \\
& \times u_{m, n}\left(x_{m}(s), y_{n}(t)\right) \mathrm{d} s \mathrm{~d} t+F_{m, n}\left(x_{m, i}, y_{n, j} ; u\right), \\
& i=1, \ldots, p ; j=1, \ldots, q
\end{aligned}
$$

$(m=0, \ldots, M-1 ; n=0, \ldots, N-1)$, where the lag term $F_{m, n}(x, y ; u)$ is defined in analogy to (2.5). For easier reference we give it explicitly: setting $(x, y):=\left(x_{m, l}, y_{n, j}\right)$ we have

$$
\begin{aligned}
F_{m, n}(x, y ; u):= & h_{m}^{(1)} \sum_{v=0}^{n-1} h_{v}^{(2)} \int_{0}^{c_{l}} \int_{0}^{1} K\left(x, y, x_{m}(s), y_{v}(t)\right) u_{m, v}\left(x_{m}(s), y_{v}(t)\right) \mathrm{d} s \mathrm{~d} t \\
& +h_{n}^{(2)} \sum_{\mu=0}^{m-1} h_{\mu}^{(1)} \int_{0}^{1} \int_{0}^{d_{j}} K\left(x, y, x_{\mu}(s), y_{n}(t)\right) u_{\mu, n}\left(x_{\mu}(s), y_{n}(t)\right) \mathrm{d} s \mathrm{~d} t \\
& +\sum_{\mu=0}^{m-1} \sum_{v=0}^{n-1} h_{\mu}^{(1)} h_{v}^{(2)} \int_{0}^{1} \int_{0}^{1} K\left(x, y, x_{\mu}(s), y_{v}(t)\right) u_{\mu, v}\left(x_{\mu}(s), y_{v}(t)\right) \mathrm{d} s \mathrm{~d} t
\end{aligned}
$$

For each pair $(m, n)(0 \leqslant m \leqslant M-1,0 \leqslant n \leqslant N-1)$, (2.9) represents a linear system in $P^{p q}$ for the unknown values $\alpha_{k, l}^{(m, n)}(k=1, \ldots, p ; l=1, \ldots, q)$ or $Y_{k, l}^{(m, n)}$ $(k=1, \ldots, p ; l=1, \ldots, q)$, depending on whether $u_{m, n}$ is expressed in the form (2.2) or (2.3). It follows from the assumed boundedness of $K$ that (2.9) has a unique solution whenever the mesh diameters

$$
h^{(1)}:=\max \left\{h_{m}^{(1)}: 0 \leqslant m \leqslant M-1\right\}, \quad h^{(2)}:=\max \left\{h_{n}^{(2)}: 0 \leqslant n \leqslant N-1\right\}
$$


are sufficiently small. Once this solution is known, the approximation $u$ is completely determined by either (2.2) or (2.3).

The computation of $u$ on $D$ proceeds recursively. However, in contrast to collocation methods for one-dimensional Volterra integral equations, there are now several different ways to implement this recursive process; we give some examples.

(i) Compute $u$ on $D_{0,0}, D_{1,0}, \ldots, D_{M-1,0}$; then on $D_{0,1}, D_{1,1}, \ldots, D_{M-1,1} ; \ldots$; finally on $D_{0, N-1}, D_{1, N-1}, \ldots, D_{M-1, N-1}$ (inner loop: $m=0, \ldots, M-1$; outer loop: $n=0, \ldots, N-1$ ).

(ii) Compute $u$ on $D_{0,0}, D_{0,1}, \ldots, D_{0, N-1}$; then on $D_{1,0}, D_{1,1}, \ldots, D_{1, N-1} ; \ldots$; finally on $D_{M-1,0}, D_{M-1,1}, \ldots, D_{M-1, N-1}$ (inner loop: $n=0, \ldots, N-1$; outer loop: $m=0, \ldots, M-1$ ).

(iii) Compute $u$ 'diagonally'; that is, on $D_{0,0} ; D_{1,0}, D_{0,1} ; D_{2,0}, D_{1,1}, D_{0,1}$; etc. In most cases we shall implement either (i) or (ii).

It is readily verified that if the collocation parameters underlying the sets $X_{m}$ and $Y_{n}$ in (2.8) are chosen so that $c_{1}=d_{1}=0$ and $c_{p}=d_{q}=1$, then the resulting collocation solution defined by (2.9) will be continuous on $D$ : $u \in S_{p, q}^{(-1)}\left(\Pi_{M, N}\right) \cap C(D)=: S_{p, q}^{(0)}\left(\Pi_{M, N}\right)$

\subsection{Global Convergence}

Let

$$
h^{(1)}:=\max \left\{h_{m}^{(1)}: 0 \leqslant m \leqslant M-1\right\}, \quad h^{(2)}:=\max \left\{h_{n}^{(2)}: 0 \leqslant n \leqslant N-1\right\},
$$

and let $h:=\max \left\{h^{(1)}, h^{(2)}\right\}$ denote the diameter of the grid $\Pi_{M, N}$. (Recall that all quantities related to the grid $\Pi_{M, N}$ depend on $M$ and/or $N$; for ease of notation this dependence is not shown explicitly.) In the following convergence analysis it will always be assumed that the underlying grid sequences are quasi-uniform; that is, there exists a finite constant $\gamma$ such that $h / \bar{h} \leqslant \gamma$ for all $M$ and $N$. Here, $\bar{h}:=\min \left\{\bar{h}^{(1)}, \bar{h}^{(2)}\right\}$, with

$$
\bar{h}^{(1)}:=\min \left\{h_{m}^{(1)}: 0 \leqslant m \leqslant M-1\right\}, \quad \bar{h}^{(2)}:=\min \left\{h_{n}^{(2)}: 0 \leqslant n \leqslant N-1\right\} .
$$

This assumption implies that

$$
h \leqslant \gamma \bar{h} \leqslant \gamma \bar{h}^{(1)} \leqslant \gamma M^{-1} X \text { and } h \leqslant \gamma \bar{h} \leqslant \gamma \bar{h}^{(2)} \leqslant \gamma N^{-1} Y
$$

hence,

$$
M N h^{2} \leqslant \gamma^{2} X Y \text { for all } M \geqslant 1, N \geqslant 1
$$

THEOREM 2.1 Assume that $g$ and $K$ in (1.1) are such that the solution $f$ of the integral (1.1) has continuous partial derivatives of order $p$ in $x$ and order $q$ in $y$ on $D$. Let $u \in S_{p, q}^{(-1)}\left(\Pi_{M, N}\right)$ denote the collocation approximation to $f$ defined by (2.9), with the underlying grid sequence $\left\{\Pi_{M, N}\right\}$ being quasi-uniform. Then the resulting collocation error $e(x, y):=f(x, y)-u(x, y)$ satisfies

$$
\|e\|_{\infty}:=\sup \{|e(x, y)|:(x, y) \in D\}=O\left(h^{\rho}\right) \quad(\text { as } M, N \rightarrow \infty),
$$


where $h$ is the grid diameter and $\rho:=\min \{p, q\}$. The above estimate holds for all collocation parameters with $0 \leqslant c_{1}<\cdots<c_{p} \leqslant 1$ and $0 \leqslant d_{1}<\cdots<d_{q} \leqslant 1$.

Proof. The above global convergence result can be verified by using a more or less straightforward generalization of techniques employed in the case of one-dimensional Volterra integral equations (see, for example, [5: pp. 251-254]; consult also [17: p. 157] for an appropriate version of Taylor's Theorem in $\mathbb{R}^{2}$ ). We omit the details; we mention, however, the two-dimensional discrete Gronwall lemma which represents the key for obtaining the above convergence order. It can be proved by induction, reducing the two-dimensional Gronwall inequality (2.11) to a sequence of one-dimensional inequalities.

LEMMA 2.1 Let $\left\{z_{m, n}\right\}$ be a sequence of non-negative numbers satisfying the inequality

$$
z_{m, n} \leqslant C_{1} h^{2} \sum_{\mu=0}^{m-1} z_{\mu, n}+C_{1} h^{2} \sum_{\mu=0}^{m} \sum_{v=0}^{n-1} z_{\mu, v}+C_{2} h^{r}
$$

$(0 \leqslant m \leqslant M-1 ; 0 \leqslant n \leqslant N-1)$, where $C_{1}$ and $C_{2}$ are positive constants and $r \in \mathbf{N}$. Then we have

$$
z_{m, n} \leqslant C_{2} h^{r} \cdot\left(1+C_{1} h^{2}\right)^{n M}\left(1+C_{1} h^{2}\right)^{m}
$$

and also

$$
z_{m, n} \leqslant C_{2} h^{r} \cdot\left(1+C_{1} h^{2}\right)^{m N}\left(1+C_{1} h^{2}\right)^{n} \quad(0 \leqslant m \leqslant M-1 ; 0 \leqslant n \leqslant N-1) .
$$

Moreover, if $M, N$, and $h$ are related by (2.10), then we obtain the uniform bound

$$
z_{m, M} \leqslant C_{2} \cdot \exp \left(C_{1} \gamma^{2} X Y\right) \cdot h^{r} \quad(0 \leqslant m \leqslant M-1 ; 0 \leqslant n \leqslant N-1) .
$$

In the context of the proof of Theorem 2.1, the exponent $r$ in (2.11) will be given by the approximation power of the spline space $S_{p, q}^{(-1)}\left(\Pi_{M, N}\right)$ for sufficiently smooth functions on $D$; that is, we have $r=\rho=\min \{p, q\}$.

\subsection{Local Superconvergence at the Grid Points}

It was shown in [3] that when one-dimensional Volterra integral equations of the second kind are solved by collocation methods using polynomial spline functions of degree $p-1$ then there are sets of collocation parameters for which the order of convergence at the grid points exceeds the order of global convergence (which is equal to $p$ ). In this section we shall show that analogous local superconvergence results hold for integral equations of the form (1.1). Further local superconvergence results will be derived in Section 3 when we consider iterated collocation approximations.

For ease of notation we shall restrict the subsequent discussion to the spline space $S_{p, p}^{(-1)}\left(\Pi_{M, N}\right)$ (that is, to the case when $p=q$ ); the modification of the corresponding local superconvergence results for the approximating space $S_{P, q}^{(-1)}\left(\Pi_{M, N}\right)$ is obvious. 
It will be convenient to associate with the collocation parameters $\left\{c_{i}\right\}$ and $\left\{d_{j}\right\}$ the functionals

$$
J_{k}^{(1)}:=\int_{0}^{1} t^{k} \cdot \prod_{i=1}^{p}\left(t-c_{i}\right) \mathrm{d} t, \quad k \in \mathbf{N}
$$

and

$$
J_{k}^{(2)}:=\int_{0}^{1} t^{k} \cdot \prod_{j=1}^{p}\left(t-d_{j}\right) \mathrm{d} t, \quad k \in \mathbf{N} .
$$

THEOREM 2.2 Assume that $g$ and $K$ in (1.1) are sufficiently smooth on their respective domains $D$ and $S$, and let $u \in S_{p, p}^{(-1)}\left(\Pi_{M, N}\right)$ denote the collocation approximation defined by (2.9), with the underlying mesh sequence being quasi-uniform. If the collocation parameters $\left\{c_{i}\right\}$ and $\left\{d_{j}\right\}$ are chosen so that the functionals in $(2.12 \mathrm{a}, \mathrm{b})$ satisfy

$$
J_{k}^{(1)}=0, \quad k=0, \ldots, r-1 ; \quad J_{r}^{(1)} \neq 0,
$$

and

$$
J_{k}^{(2)}=0, \quad k=0, \ldots, s-1 ; \quad J_{s}^{(2)} \neq 0,
$$

then the resulting collocation error, $e:=f-u$, behaves like

$$
\max _{(m, n)}\left|e\left(x_{m}, y_{n}\right)\right|=O\left(h^{\min (p+r, p+s)}\right) \quad(\text { as } M, N \rightarrow \infty),
$$

provided we have also $c_{p}=1$ and $d_{p}=1$.

If $c_{p} d_{p}<1$, then we can, in general, do no better than

$$
\max _{(m, n)}\left|e\left(x_{m}, y_{n}\right)\right|=O\left(h^{p}\right)
$$

Proof. We first note that the collocation equation (2.9) can be written in a form which is valid not only at the collocation points $C(M, N)$ but on the entire domain $D$ :

$$
u(x, y)=g(x, y)-\delta(x, y)+\int_{0}^{x} \int_{0}^{y} K(x, y, \xi, \eta) u(\xi, \eta) \mathrm{d} \xi \mathrm{d} \eta, \quad(x, y) \in D,
$$

where $\delta(x, y)$ (the residual, or defect) satisfies

$$
\delta(x, y)=0 \text { for all }(x, y) \in C(M, N)
$$

and is smooth on each subrectangle $D_{m, n}$ (provided that $g$ and $K$ are smooth functions on $D$ and $S$, respectively). Hence, subtracting (2.16) from (1.1) and setting $e=f-u$ we obtain the error equation

$$
e(x, y)=\delta(x, y)+\int_{0}^{x} \int_{0}^{y} K(x, y, \xi, \eta) e(\xi, \eta) \mathrm{d} \xi \mathrm{d} \eta, \quad(x, y) \in D .
$$


The classical theory of Volterra yields a representation of the solution of (2.18) in terms of the resolvent kernel $R(x, y, \xi, \eta)$ associated with the given kernel $K(x, y, \xi, \eta)$ (compare $[18,11,10]$ ). Accordingly, the unique solution of the integral equation (2.18) can be expressed as

$$
e(x, y)=\delta(x, y)+\int_{0}^{x} \int_{0}^{y} R(x, y, \xi, \eta) \delta(\xi, \eta) \mathrm{d} \xi \mathrm{d} \eta, \quad(x, y) \in D .
$$

We note for later reference (cf. Section 3) that the kernel $K$ and its resolvent kernel $R$ are related by the so-called resolvent equation,

$$
R(x, y, \xi, \eta)=K(x, y, \xi, \eta)+\int_{\xi}^{x} \int_{\eta}^{y} K(x, y, s, t) R(s, t, \xi, \eta) \mathrm{d} s \mathrm{~d} t,
$$

where $(x, y, \xi, \eta) \in S$.

Let now $(x, y)=\left(x_{m}, y_{n}\right)$ be a point on the grid $\Pi_{M, N}$. According to (2.19) we may write

$$
e\left(x_{m}, y_{n}\right)=\delta\left(x_{m}, y_{n}\right)+\sum_{\mu=0}^{m-1} \sum_{v=0}^{n-1} h_{\mu}^{(1)} h_{v}^{(2)} \cdot \int_{0}^{1} \int_{0}^{1} \phi_{\mu, v}^{(m, n)}(s, t) \mathrm{d} s \mathrm{~d} t,
$$

where we have set

$$
\phi_{\mu, v}^{(m, n)}(s, t):=R\left(x_{m}, y_{n}, x_{\mu}(s), y_{v}(t)\right) \cdot \delta\left(x_{\mu}(s), y_{v}(t)\right),
$$

with $x_{\mu}(s):=x_{\mu}+s h_{\mu}^{(1)}$ and $y_{v}(t):=y_{v}+t h_{v}^{(2)}$. Note that, by the definition of the residual $\delta$ the integrands $\phi_{\mu, r}^{(m, n)}(s, t)$ vanish for all $(s, t)=\left(c_{i}, d_{j}\right)$ (recall (2.17)).

Consider a typical integral in (2.21). If it is approximated by an interpolatory quadrature rule using as abscissae the points of the rectangular array $\left\{\left(c_{i}, d_{j}\right): 1 \leqslant i \leqslant p, 1 \leqslant j \leqslant p\right\}$ and possessing the weights

$$
w_{l, j}:=\int_{0}^{1} \int_{0}^{1} L_{i, j}(s, t) \mathrm{d} s \mathrm{~d} t, \quad i=1, \ldots, p ; j=1, \ldots, p
$$

(cf. (2.3)), then, letting $E_{\mu, \vartheta}^{(m, n)}$ denote the corresponding quadrature error, we have

$$
\int_{0}^{1} \int_{0}^{1} \phi_{\mu, v}^{(m, n)}(s, t) \mathrm{d} s \mathrm{~d} t=\sum_{i=1}^{p} \sum_{j=1}^{p} w_{l, j} \phi_{\mu, v}^{(m, n)}\left(c_{i}, d_{j}\right)+E_{\mu, v}^{(m, n)}
$$

Since $\phi_{\mu, v}^{(m, n)}\left(c_{i}, d_{j}\right)=0$ for $i=1, \ldots, p ; j=1, \ldots, p$, the above expression reduces to

$$
\int_{0}^{1} \int_{0}^{1} \phi_{\mu, v}^{(m, n)}(s, t) \mathrm{d} s \mathrm{~d} t=E_{\mu, v}^{(m, n)} \quad(0 \leqslant \mu<m \leqslant M-1,0 \leqslant v<n \leqslant N-1) .
$$

Hence, (2.21) may be replaced by

$$
e\left(x_{m}, y_{n}\right)=\delta\left(x_{m}, y_{n}\right)+\sum_{\mu=0}^{m-1} \sum_{v=0}^{n-1} h_{\mu}^{(1)} h_{v}^{(2)} E_{\mu, v}^{(m, n)}
$$


( $m=1, \ldots, M-1 ; n=1, \ldots, N-1)$. This last equation reveals that the order of the collocation error at the grid points is governed by the orders of the residual on the grid $\Pi_{M, N}$ and the quadrature errors induced by the interpolatory tensor product quadrature rules for the lattice defined by the points $x_{m}+c_{i} h_{m}^{(1)}$ and $y_{n}+d_{j} h_{n}^{(2)}(c f .(2.7 \mathrm{a}, \mathrm{b}))$. The assumed orthogonality conditions $(2.13 \mathrm{a}, \mathrm{b})$ imply that the one-dimensional, interpolatory $p$-point quadrature rules which have abscissae based on the collocation parameters $\left\{c_{i}\right\}$ and $\left\{d_{j}\right\}$ have, respectively, degrees of precision $p+r-1$ and $p+s-1$ (compare, for example, [6]; see also $[1,12,17])$. Hence, the quadrature errors $E_{\mu, v}^{(m, n)}$ in (2.24) all satisfy

$$
\left|E_{\mu, v}^{(m, n)}\right| \leqslant C h^{\min (p+r, p+s)}
$$

for some finite constant $C$ (note that, by (2.20), the resolvent kernel $R$ inherits the smoothness properties of the given kernel $K$, while the residual $\delta$ is, according to (2.16), smooth on each subdomain $D_{m, n}$ ).

Consider first the case where $c_{p}=1$ and $d_{p}=1$. This choice of the collocation parameters implies that $\left(x_{m}, y_{n}\right)$ in (2.24) is a collocation point, and thus we have $\delta\left(x_{m}, y_{n}\right)=0$. It follows from (2.24), using the result $M N h^{(1)} h^{(2)} \leqslant M N h^{2} \leqslant$ $\gamma^{2} X Y<\infty$ (cf. (2.10)), that the assertion (2.14) holds. If the collocation parameters are chosen such that $c_{p} d_{p}<1$, then $\left(x_{m}, y_{n}\right)$ will not belong to the set $C(N, N)$ of collocation points. It can be shown that in this case the residual will, in general, converge no faster than $\delta\left(x_{m}, y_{n}\right)=O\left(h^{p}\right)$. Hence, by (2.24),

$$
\max \left|e\left(x_{m}, y_{n}\right)\right|=O\left(h^{p}\right)+O\left(h^{\min (p+r, p+s)}\right)=O\left(h^{p}\right),
$$

regardless of the choice of the other collocation parameters. This proves (2.15).

The following corollary spells out the local superconvergence orders for some practically relevant choices of the collocation parameters. Analogous results for sets $\left\{c_{i}\right\} \neq\left\{d_{i}\right\}$ are obvious.

COROLlary 2.1 (a) If the collocation parameters $\left\{c_{i}\right\}$ are given by the Radau II points (that is, by the zeros of $P_{p}(2 t-1)-P_{p-1}(2 t-1)$, where $P_{p}(2 t-1)$ is the shifted Legendre polynomial of degree $p$ ), and if $d_{j}=c_{j}, j=1, \ldots, p$, then the collocation error satisfies

$$
\max _{(m, n)}\left|e\left(x_{m}, y_{n}\right)\right|=O\left(h^{2 p-1}\right) \text {. }
$$

(b) If the collocation parameters $\left\{c_{i}\right\}$ are the Lobatto points (that is, the zeros of $\left.t(t-1) P_{p-1}^{\prime}(2 t-1)\right)$, and if $d_{j}=c_{j}, j=1, \ldots, p$, then

$$
\max _{(m, n)}\left|e\left(x_{m}, y_{n}\right)\right|=O\left(h^{2 p-2}\right) \quad(p \geqslant 2) .
$$

Here, the collocation approximation $u$ is in $S_{p, p}^{(0)}\left(\Pi_{M, N}\right)$.

(c) The choice of the Gauss points as the collocation parameters (that is,

$$
\left.P_{p}\left(2 c_{i}-1\right)=P_{p}\left(2 d_{i}-1\right)=0, \quad i=1, \ldots, p\right)
$$

does not, in general, lead to local superconvergence at the grid points: we obtain

only.

$$
\max _{(m, n)}\left|e\left(x_{m}, y_{n}\right)\right|=O\left(h^{p}\right)
$$


The proof of these results is straightforward: it makes use of the orthogonality conditions $(2.13 \mathrm{a}, \mathrm{b})$ (where we have, respectively, $r=s=2 p-1 ; r=s=2 p-2$; and $r=s=2 p$; here, the Gauss points satisfy $c_{p}<1$ and $d_{p}<1$ ) and the resulting degrees of precision of the quadrature formulae based on these collocation parameters.

In the next section we shall show that subjecting the collocation approximation with respect to the Gauss points to a single iteration yields local superconvergence of order $2 p$ at the grid points.

\section{Iterated collocation approximations}

Let $u \in S_{p, q}^{(-1)}\left(\Pi_{M, N}\right)$ be the collocation approximation generated by (2.9). The corresponding iterated collocation approximation $u^{I}$ is defined by

$$
u^{I}(x, y):=g(x, y)+\int_{0}^{x} \int_{0}^{y} K(x, y, \xi, \eta) u(\xi, \eta) \mathrm{d} \xi \mathrm{d} \eta, \quad(x, y) \in D .
$$

Note that $u^{I}$ coincides with $u$ at all collocation points, and we have $u^{I} \in C(D)$ for any choice of the collocation parameters.

THEOREM 3.1 Assume that $g$ and $K$ in (1.1) are $2 p$ times continuously differentiable on $D$ and $S$, respectively, and let $u \in S_{p, p}^{(-1)}\left(\Pi_{M, N}\right)$ be the collocation approximation given by (2.9), where the collocation parameters $\left\{c_{i}\right\}$ are the Gauss points and $d_{i}=c_{i}(i=1, \ldots, p)$. If $u^{l}$ is the corresponding iterated collocation approximation defined by (3.1), then its error, $e^{l}:=y-u^{l}$, satisfies

$$
\max _{(m, n)}\left|e\left(x_{m}, y_{n}\right)\right|=O\left(h^{2 p}\right) \quad(\text { as } M, N \rightarrow \infty) .
$$

Proof. It follows from (1.1) and (3.1) that $e^{\prime}$ is given by

$$
e^{I}(x, y)=\int_{0}^{x} \int_{0}^{y} K(x, y, \xi, \eta) e(\xi, \eta) \mathrm{d} \xi \mathrm{d} \eta, \quad(x, y) \in D .
$$

Using the expression (2.19) for the collocation error, and recalling the resolvent equation (2.20), we are led to

$$
e^{I}(x, y)=\int_{0}^{x} \int_{0}^{y} R(x, y, \xi, \eta) \delta(\xi, \eta) \mathrm{d} \xi \mathrm{d} \eta, \quad(x, y) \in D,
$$

where $\delta$ is the residual introduced in (2.16). This expression is reminiscent of (2.19), except that now the free term $\delta(x, y)$ no longer occurs. Hence, the arguments we applied to (2.21) to establish the local superconvergence results of Theorem 2.2 can be employed in an identical manner: since in (2.24) the term $\delta\left(x_{m}, y_{n}\right)$ is now missing, the quadrature errors $E_{\mu, v}^{(m, n)}$ are now those for the $p \times p$ tensor-product Gauss quadrature formulae $[1,17]$, and we have $\left|E_{\mu, v}^{(m, n)}\right| \leqslant C h^{2 p}$ for some finite constant $C$. The result (3.2) follows immediately because $M N h^{2} \leqslant \gamma^{2} X Y<\infty($ see $(2.10))$.

It can be shown that if $(x, y)$ is not a grid point then the iterated collocation 
error converges only like $e^{\prime}(x, y)=O\left(h^{p}\right)$. This is in striking contrast to the situation encountered when solving two-dimensional Fredholm integral equations by collocation and iterated collocation in $S_{p, p}^{(-1)}\left(\Pi_{M, N}\right)$ : here, collocation at the Gauss points yields $e^{I}(x, y)=O\left(h^{2 p}\right)$ for all $(x, y) \in D$ (compare [9]).

\section{Numerical examples}

In order to obtain a computational form of the collocation equation (2.9) and the expression (3.1) for $u^{I}\left(x_{m}, y_{n}\right)$ we have to carry out a further discretization step. This involves the approximation of the integrals in (2.9), (2.9'), and (3.1) (in the last case over the subdomains $D_{\mu, v}$ ) by appropriate quadrature processes. If these quadrature processes employ tensor-product quadrature formulae whose $p \times p$ abscissae are based on the $p \times p$ collocation parameters $\left\{c_{i}\right\}$ and $\left\{d_{j}\right\}$ (assuming that the approximating space is $S_{p, p}^{(-1)}\left(\Pi_{M, N}\right)$ ), then it is easy to show (along the lines of [3]) that the results given in Theorem 2.2, Corollary 2.1, and Theorem 3.1 remain valid for the discretized collocation and iterated collocation method.

We illustrate the numerical performance of these discretized methods by solving (1.1), with $D=[0,2] \times[0,2]$,

$$
g(x, y)=\exp (-a x)\left(\cos (b y)-\frac{c x}{2 b}(\sin (b y)+b y \cdot \cos (b y))\right),
$$

and

$$
K(x, y, \xi, \eta)=c \cdot \exp (-a(x-\xi)) \cdot \cos (b(y-\eta)),
$$

in the spline space $S_{2,2}^{(-1)}\left(\Pi_{m, N}\right)(p=q=2)$. The collocation parameters are $c_{1}=d_{1}=(3-\sqrt{ } 3) / 6, \quad c_{2}=d_{2}=(3+\sqrt{ } 3) / 6$, and hence the quadrature formulae employed in (2.9), $\left(2.9^{\prime}\right)$, and (3.1) (with $(x, y)$ replaced by $\left(x_{m}, y_{n}\right)$ ) are tensor products of two-point Gaussian rules. According to Theorem 2.2 we expect the collocation error to behave like $e\left(x_{m}, y_{n}\right)=O\left(h^{2}\right)$, while (Theorem 3.1) $e^{l}\left(x_{m}, y_{n}\right)=O\left(h^{4}\right)$.

In Tables 4.1 and 4.2 we exhibit a sample of collocation and iterated collocation errors corresponding to the parameter values $a=b=1, c=-1$; the exact solution of the integral equation is given by $f(x, y)=\exp (-a x) \cos (b y)$.

Although the mesh diameters are still quite large $\left(h^{(1)}=h^{(2)}=h=2 / M\right)$, the resulting piecewise bilinear collocation approximations and their iterates are

TABLE 4.1

Collocation errors

\begin{tabular}{rcccc}
\hline$M=N$ & $|e(1,1)|$ & $|e(2,1)|$ & $|e(1,2)|$ & $|e(2,2)|$ \\
\hline 4 & $1 \cdot 464 \mathrm{D}-4$ & $1 \cdot 823 \mathrm{D}-4$ & $1 \cdot 665 \mathrm{D}-3$ & $5 \cdot 565 \mathrm{D}-4$ \\
8 & $2 \cdot 143 \mathrm{D}-5$ & $2 \cdot 670 \mathrm{D}-5$ & $1.932 \mathrm{D}-4$ & $6 \cdot 124 \mathrm{D}-5$ \\
16 & $3 \cdot 673 \mathrm{D}-6$ & $3 \cdot 539 \mathrm{D}-6$ & $2 \cdot 307 \mathrm{D}-5$ & $7 \cdot 100 \mathrm{D}-6$ \\
\hline
\end{tabular}


TABLE 4.2

Iterated collocation errors

\begin{tabular}{rllll}
\hline$M=N$ & $\left|e^{\prime}(1,1)\right|$ & $\left|e^{\prime}(2,1)\right|$ & $\left|e^{\prime}(1,2)\right|$ & $\left|e^{\prime}(2,2)\right|$ \\
\hline 4 & $4 \cdot 143 \mathrm{D}-5$ & $1 \cdot 036 \mathrm{D}-5$ & $4 \cdot 911 \mathrm{D}-5$ & $1 \cdot 913 \mathrm{D}-5$ \\
8 & $2 \cdot 554 \mathrm{D}-6$ & $6 \cdot 349 \mathrm{D}-7$ & $3 \cdot 019 \mathrm{D}-6$ & $1 \cdot 174 \mathrm{D}-6$ \\
16 & $1 \cdot 594 \mathrm{D}-7$ & $3 \cdot 959 \mathrm{D}-8$ & $1 \cdot 883 \mathrm{D}-7$ & $7 \cdot 325 \mathrm{D}-8$ \\
\hline
\end{tabular}

rather accurate: while the values of $u$ seem to converge somewhat more rapidly than predicted by Theorem 2.2 (cf. (2.15)), the values in Table 4.2 confirm the predicted order of local superconvergence, $O\left(h^{4}\right)$, of Theorem 3.1.

\section{Concluding remarks}

The present study of collocation methods for two-dimensional Volterra integral equations (1.1) was motivated in part by the Darboux problem for hyperbolic differential equations of the form

$$
z_{x y}=G(x, y, z), \quad(x, y) \in D,
$$

where $D:=\left\{(x, y) \in \mathbb{R}^{2}: 0 \leqslant x \leqslant X, 0 \leqslant y \leqslant Y\right\}$. The values of the solution $z=z(x, y)$ are prescribed on part of the boundary of $D$ :

$$
z(x, 0)=g_{1}(x), \quad 0 \leqslant x \leqslant X ; \quad z(0, y)=g_{2}(y), \quad 0 \leqslant y \leqslant Y
$$

(compare also [7]). The given functions $g_{1}$ and $g_{2}$ are assumed to satisfy $g_{1}(0)=g_{2}(0)$. This problem is equivalent to the Volterra integral equation

$$
z(x, y)=g(x, y)+\int_{0}^{x} \int_{0}^{y} G(\xi, \eta, z(\xi, \eta)) \mathrm{d} \xi \mathrm{d} \eta, \quad(x, y) \in D,
$$

where the non-homogeneous term $g(x, y)$ contains the given initial values

$$
g(x, y)=g_{1}(x)+g_{2}(y)-g_{1}(0)
$$

There are a number of advantages in solving (5.1) rather than the original differential equation: the collocation approach described in this paper allows for the construction of numerical schemes of arbitrarily high order (while the derivation of high-order finite-difference methods for the direct solution of the problem is more difficult and dependent on the availability of sufficiently accurate starting values). This approach to the Darboux problem will be discussed elsewhere, including the case where the given boundary data is non-smooth (compare also [9], where the attainable order of convergence of spline collocation methods for two-dimensional Fredholm integral equations with non-smooth data is studied).

\section{Actnowledgement}

The work of the first author was supported in part by the Natural Sciences and Engineering Research Council of Canada (NSERC Grant A9406). 


\section{REFERENCES}

1. Ahlin, A. C. 1962 On error bounds for Gaussian cubature. SIAM Rev. 4, 25-39.

2. Bel tyukov, B. A., \& Kuznechikhina, L. N. 1976 A Runge-Kutta method for the solution of two-dimensional nonlinear Volterra integral equations. Diff. Eqns 12, 1169-1173.

3. BrUNNER, H. 1984 Iterated collocation methods and their discretizations for Volterra integral equations. SIAM J. numer. Anal. 21, 1132-1145.

4. BRUNNer, H. 1987 Collocation methods for one-dimensional Fredholm and Volterra integral equations. Proceedings 'The State of the Art in Numerical Analysis', Birmingham 1986 (eds A. Iserles \& M. J. D. Powell). Oxford: University Press, $563-600$.

5. Brunner, H., \& VAN DeR Houwen, P. J. 1986 The Numerical Solution of Volterra Equations. Amsterdam: North-Holland.

6. Davis, P. J., \& RabinowtTZ, P. 1984 Methods of Numerical Integration, 2nd edition. New York: Academic Press.

7. Dobner, H.-J. 1987 Bounds for the solution of hyperbolic problems. Computing 38, 209-218.

8. Graham, I. G. 1985 Numerical methods for multidimensional integral equations. Computational Techniques and Applications (eds J. NoYe \& C. Fletcher). Amsterdam: North-Holland, 335-351.

9. JOE, S. 1985 The numerical solution of second kind Fredholm integral equations. Ph.D. Thesis, University of New South Wales, Kensington.

10. Kauthen, J.-P. 1986 Résolution numérique des équations intégrales de Volterra dans $\mathbf{R}^{2}$. Diploma Thesis, University of Fribourg.

11. Lalesco, T. 1912 Introduction a la Théorie des Équations Intégrales. Paris: A. Hermann \& Fils.

12. LETHER. F. 1970 An error representation for product cubature rules. SIAM J. numer. Anal. 7, 363-365.

13. Prenter, P. M. 1975 Splines and Variational Methods. New York: Wiley.

14. Schumaker, L. L. 1981 Spline Functions: Basic Theory. New York: Wiley.

15. Schumaker, L. L. 1984 On spaces of piecewise polynomials in two variables. Approximation Theory and Spline Functions (eds S. P. SinOH, J. W. H. BURRY, \& B. WATSON). Dordrecht: Reidel, 151-197.

16. SingH, P. 1976 A note on the solution of two-dimensional Volterra integral equations by splines. Indian J. Math. 18, 61-64.

17. STROUD, A. H. 1971 Approximate Calculation of Multiple Integrals. Englewood Cliffs: Prentice-Hall.

18. Volterra, V. 1896 Sulla inversione degli integrali multipli. Atti Accad. Naz. Lincei Rend. Cl. Sci. Fis. Mat. Natur. (5) 5, 289-300. 
